\title{
INT. JOURNAL OF COMPUTER INTEGRATED MANUFACTURING
}

\section{Smart CPS: Vertical Integration Overview and User Story with a Cobot}

\author{
Levente Tamas ${ }^{\mathrm{a}}$ and Mircea Murar ${ }^{\mathrm{b}}$ \\ ${ }^{a}$ Robotics and Nonlinear Research Group, Technical University of Cluj-Napoca, Memorandumului st \\ 28, Cluj-Napoca, Romania; ${ }^{b}$ Design Engineering and Robotics , Faculty of Machine Building, \\ Technical University of Cluj-Napoca, 103-105 Boulevard, Cluj-Napoca, Romania
}

\author{
ARTICLE HISTORY \\ Compiled November 28, 2018
}

\begin{abstract}
In this paper an overview and a demonstration for the vertical integration of manufacturing enterprise layers are described by implementing the Manufacturing Execution System (MES). In the first part of the paper the details regarding the MES implementation are described, while in the second part the use case specific insights are highlighted. The presented use case contains each important step of a production line involving also a collaborative Baxter type robot and the state-of-the-art tools for MES implementation. The cobot-involved use case is relevant and generic enough in the context of Industry 4.0 offering a good overview of a typical vertical integration use case which can be generalized and applied to manufacturing scenarios. The paper ends with the lessons learned from the vertical integration process as well as future direction which can be followed in such a context.
\end{abstract}

\section{KEYWORDS}

Smart CPS, MES, cobot, Industry 4.0.

\section{Introduction}

Today's manufacturers are subject to the pressure to achieve and maintain high industrial performance dealing with short production life cycles as well as sever environmental regulations for sustainable production. The cause of this conflict mainly originates from the lack of vertical integration of different components inside a company, in order to achieve evolving manufacturing systems.

A possible way to achieve the vertical integration is through the coherent usage of the Manufacturing Execution Systems (MES) and the Enterprise Resource Planning (ERP) in a common automated information flow in order to achieve smart, safe and sustainable $(3 \mathrm{xS})$ production in modern cyber-psychical systems (CPS).

The automatic processing of information can benefit from emerging technologies such as the Internet of Things (IoT), embedded systems, cyber physical systems (CPS), cloud computing, and remote access. These are disruptive technologies which are already suggested in the de facto standard Industry 4.0 German strategic plan for the industry of the future (Kagermann et al.|2013), (Uhlmann et al.|2017). Although similar initiatives for the next generation manufacturing standardization exist in other countries, such as Internet+ in China or the Industrial Internet Consortium, the popularity of Industry 4.0 grows fast both in the research and

Correpsonding author: Mircea.Murar@muri.utcluj.ro

L. Tamas is partially affiliated with Pannon University from Veszprem, Hungary 
applied sciences communities (Weyer et al. 2015; Uhlmann, Hohwieler, and Geisert 2017). This is mainly due to leveraging existing standards such as the ISA-88 for batch processing or the ISA-95 for enterprise control beside the well-established OPC Unified Architecture (UA) and PLCopen ones (Meier and Rauschecker 2015).

In addition, the success of Industry 4.0 strategic plan is also due to promptly accepting new challenges such as the usage of collaborative worker within augmented reality(AR) enabled environments containing collaborative robots (cobots) (Militaru, Mezei, and Tamas 2016). This is one step ahead the traditional way of thinking about cobots (such as the fact that they may replace humans) and it takes the Human-Robot-Interaction/Interface (HRI) to another level. According to (Berger and Wahrendorff 2016) in the H2020 horizon, the fastest growth in the robotics market is predicted for cobot-like devices compared to unmanned aerial vehicles, autonomous agents, or AR devices. Thus, the cobot and its vertical integration in production by implementing a MES layer is an important topic for both for robotics research dealing with HRI topics and for the manufacturing science. These open up new challenges such as the integration of cobots in real life applications.

In this paper the authors propose a use case to highlight the mile-stones of vertical integration in a production chain by implementing MES while using a Baxter type cobot. The purpose of this pilot project is generalizable to larger/more complex scenarios, containing all the necessary components of a generic use case. A thorough review of the existing solution in this emerging field is also summarized in a table containing the most relevant solutions currently available for MES.

\subsection{Motivation}

A reduced number of research papers focused on vertical integration of enterprise layers are available in academic environment (Arica and Powell 2018). An early specification for the MES dates back to the 90's in the works of (Barkmeyer et al. 1999; Deuel 1994). Some of the first reviews in this domain are presented in the work of (De Ugarte, Artiba, and Pellerin 2009). As a consequence, there is an urgent need for use cases that highlight the tools, stages and methods required to migrate from conventional manufacturing technologies to emerging ones. Such use cases should also focus on the implementation details, in order to bring forward the main characteristics of this approach.

\subsection{Related work}

Recently, the number of scientific reports dealing with MES implementation (Weyer et al. 2015; Wang et al. 2016), vertical integration (Jung et al. 2017; Morariu, Borangiu, and Raileanu 2015) and Industry 4.0 (Kagermann et al.2013) has grown impressively.

A good overview of the past, current and future trends in the manufacturing domain can be found in (Kang et al. 2016a), while details regarding the standards related to this domain are presented in (Lu, Morris, and Frechette 2016). As this domain is strongly connected to the ICT and cyber-psychical systems (Monostori 2014; Lee, Bagheri, and Kao|2015), related domains such as robotics with new paradigms (Bevilacqua et al.2015) are also represented. The impact of MES systems on the labor force is presented in the early work of (Nasarwanji et al. 2009).

Closely related to the scope of this paper are the works of (Zarte et al. 2016), while solutions for small lot production systems can be found in (Perzylo et al. 2016). A small scale use case is reported in the work of (Arab-Mansour, Millet, and Botta-Genoulaz 2017) which analyzes the business impact of the proposed solution. 


\subsection{Contributions}

A straightforward demo application is proposed for the MES vertical integration using stateof-the-art tools alongside the integration of a dual-arm collaborative robot (RethinkRobotics 2018) and an AGV into the Industry 4.0 concept. By doing this, the necessary steps for the MES implementation in a generic way are covered, which can serve as starting point for more complex scenarios. The proposed work flow, graphically represented in Figure 1, is easy to follow as a number of light bulbs, specified by the end user (in the manufacturing enterprise interface), are handled in the production, in the quality check and in the deployment chain.

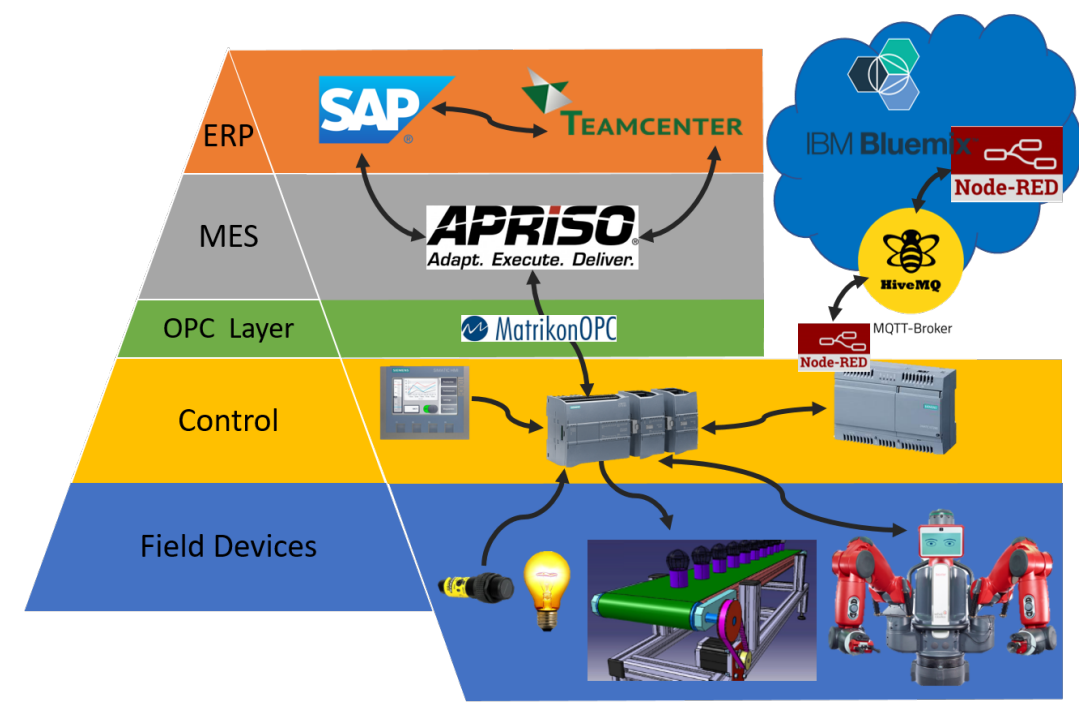

Figure 1.: Hierarchical overview of the MES server implementation from the shop floor with cobot to the ERP with cloud solutions

The main technical contributions consist of the implementation of the whole vertical integration of the custom use case, including a cobot, into an existing MES system in a company. This integration contains novel solutions such as the use of an open source (ROS) based cobot and AGV which enable a flexible, easily reconfigurable workspace as well as a new collaborative human-robot approach to manufacturing. In order to achieve this, state-of-the-art 3D perception techniques were used to observe the workspace of the robot and flexible, dynamic obstacle aware planning algorithms were implemented to ensure safe operation near the human operator (Militaru, Mezei, and Tamas 2017). The integration of both the cobot and the custom conveyor belt into the commercial MES requires in-house system and software design and development, presented in Section 3 .

In terms of key process indicators(KPI), the proposed vertical integration helps to overcome some of the major client side challenges such as: rigid sensor infrastructure (the workspace of the robot can be reorganized in a flexible manner), reactive decision making (the lack of real-time data analysis was solved with the data being available from all levels within the MES with Apriso), and the need for custom human-machine-interface (realized with innovative solutions within robotics for the Baxter cobot/AGV). In quantitative terms, the proposed solution enhances the workers' productivity by more than $20 \%$, increases the waste ratio by over $15 \%$ and enhances the overall equipment efficiency rate by $10 \%$ compared to the solution without flexible setup and without real-time data analysis. 


\section{Current state-of-the-art for MES}

To identify the state-of-the-art in a field driven by emerging technologies a thorough state-ofthe-art research is needed covering both academical research papers as well as white paper industrial reports.

Therefore, to obtain a holistic overview of the field, this section was divided into several subsections dealing with: general facts, definition and architecture of MES, a chronological approach and report on literature, a summary and comparison of commercially available MES solutions and an overview of MES key performance indicators.

\subsection{General facts, definition and architecture}

Manufacturing information and execution systems (MIES) emerged during 1990s mostly as internal developments of enterprises to support their production management procedures. These developments marked the beginning of what these days is know as MES (Hawker 1999).

MES were deployed to bridge and integrate ERP and physical operational systems to achieve a fully automated and integrated manufacturing management according to (Huang 2002) and (Choi and Kim 2002).

Several MES reference models were developed by manufacturing related organization like: MESA, ISA, VDI, PERA and NAMUR to stand as a common point for MES development (Sauer 2010), (Lasi 2013), (Lamparter et al. 2011) and (Naedele et al. 2015).

The reference model developed by MESA in 1997 is one of the most widely used (MESA 1997). It defines the functional areas of a MES: labor management resource allocation, dispatching, product tracking, quality management, performance analysis, process management, scheduling, document control and maintenance management (Naedele et al.2015). The scope of previously stated functional areas and the way how information should flow in a MES are detailed in ISA-95 standard (Naedele et al.2015; Cupek et al.2016).

ISA-95 standard highlights MES place in an enterprise and describes its tasks. Figure 3 presents a vertical integration of a manufacturing enterprise levels using a MES.

Level 0, also known as the shop-floor, represents the process of goods production itself. Level 1 contains all the sensors and actuators used to sense and interact with the shop-floor. Level 2 consist of control and monitor equipment like: PLCs, HMIs, distributed control systems (DCS), SCADA and other advanced manufacturing resources. On this level process related data is acquired at a high sampling rate from the process, furthermore analyzed, logged and visualized by specific manufacturing resources. A custom developed control logic runs inside control equipment to provide the required technological functionalities of the shopfloor.

MES is placed on level 3 and acts as a bridge between level 2 and level 4. Level 3 receives business related information from level 4 and transposes it such that it can be parsed by control equipment at level 2 to control the process. In a similar way, level 3 gets process related information from level 2 and transposes it in such way that this can be understood by enterprise management at level 4 so further decisions based on this information can be made.

Considering the emergence of cloud computing, level 1 to 4 within a manufacturing enterprise can be connected to cloud services and advanced functionalities for monitoring, control, data analytics, artificial intelligence can be used to add value within the manufacturing supply chain. 


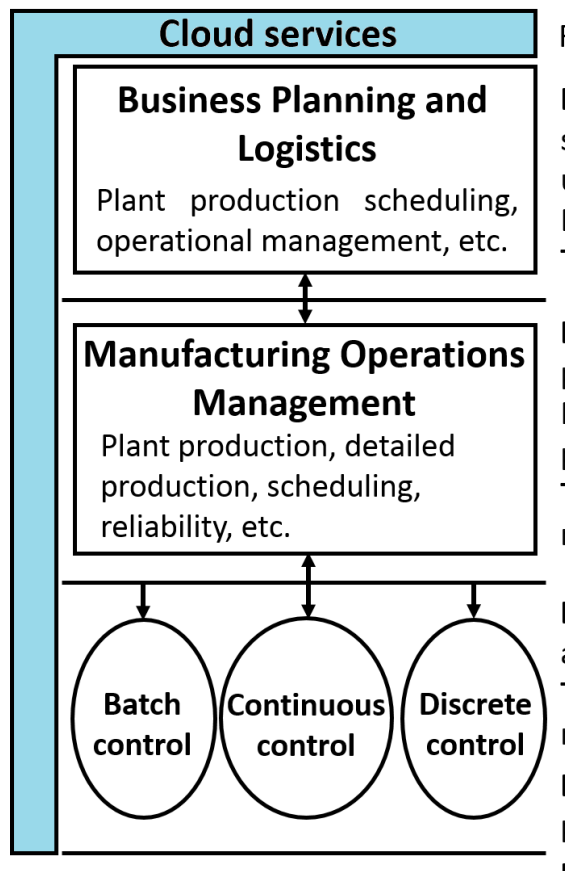

Remote services and functionalities

L4. Establishing the basic plant schedule-production, material use, delivery and shipping. Determining inventory levels. Time frame: months, weeks, days

L3. Work flow/recipe control to produce the desired products. Maintain records and optimize the production process in real-time. Time frame: days, shifts, hours, minutes, seconds

L2. Monitoring, supervisory control and automated control of process. Time frame: minutes, seconds, milliseconds, microseconds.

L1. Sensing and manipulating the production processes.

LO. The actual production process

Figure 2.: Vertical integration of ISA95, ISA-88 and cloud services

\subsection{Chronological review of MES}

The literature search for MES related research articles was focused upon the following keywords: manufacturing execution system, vertical integration, ISA-95, digitalization, Industry 4.0, enterprise resource planning and manufacturing execution system key performance indicators and specific filters. Even though, such a great variety of keywords were used to search research articles in the literature, the lack of MES related articles was observed.

The scarcity of MES literature is also highlighted by (Arica and Powell|2018) which identifies only one literature review since 2009 done by (De Ugarte, Artiba, and Pellerin 2009) which is focused on technological developments and disadvantages of MES. A second literature review in the field is done by (Arica and Powell 2018) with the goal of supporting the MES selection process. The approach proposes a taxonomy to characterizes MES in two categories of factors: business and manufacturing and technological.

In order to obtain a glimpse on MES trends, this paper uses a chronological approach to summarize the evolution in this domain focusing on the used technologies and application domains.

\subsubsection{Until 1999}

One of the first attempts to guide in a standardized fashion the development of MIES was SEMATECH CIM (Hawker 1999). Built on common object request broker architecture (CORBA), a standard used to achieve interoperability between distributed systems (CORBA n.d.). SEMATECH CIM was a standardized framework developed as a solution to the obsolete and inflexible custom built MIES to implement interoperability under a standardized approach (Cheng et al. 1999). 


\subsubsection{From 2000 until 2004}

Considering geographically distributed manufacturing facilities an approach based on a collaborative work-flow-based protocol (CWP) was proposed by (Huang 2002). The CWP integrates Internet technology and expert systems to achieve connectivity with data acquisition and control units of distributed MES and support decision process.

To integrate a shop floor and a flexible manufacturing systems (FMS) into an ERP system, (Choi and Kim 2002) uses the functional modeling methodology (IDEF0) to analyze and describe the manufacturing functions needed by a MES as an intermediate component between these two. Considering the reduced number of commercially available MES solutions and their limitations, a two-tier MES architecture was developed using Visual Studio and Oracle DBMS. Main-MES is directly connected to ERP systems while managing the entire shopfloor and the FMS-MES.

(Canche et al.2004) describes in details the disadvantages of the technologies involved in the architecture proposed by (Cheng et al.|1999) and (Choi and Kim 2002) while highlighting the advantages and potential of the latest ethnologies. Data sharing between different systems in an enterprise and integration of new software packages could benefit from adoption of web services, self-describing software, XML language, Internet standards and protocols.

(Cheng, Chang, and Wu 2004) argues that previously MES architecture are hard to reconfigure, difficult to modify and they lack two important properties: failure recovery and security certification. As a solution to the identified disadvantages is considered the advantages of the holon and holarchy concepts which propose a systematic methodology to develop Holonic MES.

\subsubsection{From 2005 until 2009}

(Li et al. 2005) analyzes the architecture of a MES in an iron and steel enterprise to emphasize the importance of vertical integration for the value added in the enterprise.

(Simo, Stadzisz, and Morel 2006) develops a design and simulation tool, namely ANALYTICE II, to allow simulation and testing of product driven Holonic MES based on smart products and entities that are responsible for driving the production.

(Verstraete et al.2008) achieves within a simulation environment the cooperation between a HMES developed on PROSA architecture (Van Brussel et al. 1998) and a planning system. Several improvements for HMES architecture are presented based on simulation results. (Valckenaers et al. 2007) improves previous research by implementing an automatic schedule execution in decentralized HMES. Therefore, allowing HMES to generate solutions to different unplanned events which are not related to a given schedule.

(Pascal and Demongodin 2008) reports a partial implementation in an automotive related industry of a product centered HMES developed using JADE multi-agent system and integration with ERP. Messages exchanged between holons use XML format to describe their role and are transferred via a TCP/IP connection.

(Panetto and Molina 2008) foresees integration of semantic web services as a key approach to align the diversity of enterprises, manufacturing systems and equipment towards manufacturing enterprise integration and interoperability.

\subsubsection{From 2010 until 2014}

(Lamparter et al. 2011) identifies, that most of disruptive events in a supply chain managements (SCM) are operated at SCM and ERP level between globally connected enterprises. Reactive mechanisms and triggered actions lack detailed information and real-time control over the on-going production processes. To efficiently solve disruptive events on production level 
an event based production order optimization was developed on top of a MES. To optimize product order the MES framework is extended with an electronic product code information service (EPCIS) compliant event processing mechanism able to adjust production schedule considering the severity of the event. The proposed solution can optimize the tracking and tracing information in production planning and control layers. Using EPCIS mechanisms for ERP and SCM, the proposed solution adapts to the production processes to events happening at the SCM level. The solution was implemented in Siemens SmartAutomation lab by using Siemens state-of-the-art MES software (Lamparter et al. 2011).

A good overview of multi-agent architectures used to design MES is presented in (Roln and Martnez 2012). To enhance previous researches a new MES architecture featuring autonomy, stability and disturbance rejection was proposed, design, simulated and tested.

(Wang et al. 2012) reports achieving real-time control of the shop-floor in a production system from mold and die industry by implementing an RFID based MES. (Dai et al. 2012) reports similar results in automotive industry.

(Ksksal and Tekin 2012) provides information regarding a MES implemented in aerospace industry. e-Factory support full traceability of issued documents and manufacturing process operations.

Zhong presents a development of a MES in the field of mass customization production based on RFID technology. Developed MES deals with uncertainty and complexity of shopfloor generated by a wide variety of product components and inefficient scheduling due to manual data collection (Zhong et al. 2013).

(Gonalves and Filho 2013) presents the deployment of Siemens MES solution, into alcohol industry to deal with increased productivity and overhead reduction. Information from decisions taken at top management level were integrated in such a way to optimize the overall production process.

(Groger et al.2013) argues that ERP and MES are top-level management centered designed and as a result a limited quantity of useful information is delivered from MES to workers. In such conditions dead-times, inflexibility and lack of adaptability can be experienced. As a solution, an operational process dashboard is proposed to empower shop floor workers with important information to optimize the production process.

\subsubsection{From 2015 until present}

Vertical integration of the enterprise horizontally distributed layers is the key towards highly adaptive manufacturing enterprises. This is especially true today thus it is one of Industry 4.0 core enabler.

(Weyer et al. 2015) presents one of the fewest vertical integration of the information systems that can be found in an enterprise with the shop floor as a result of an EU funded project named SmartFactoryKL. The research and developments made a step towards Industry 4.0 and enterprise integration in a decentralized fashion by developing a standardized plug and play multi-vendor interface. The project uses technologies like: RFID, web services and OPC UA to achieve vertical integration. As mentioned in the paper, the key to success was due to the coordinated standardization and representation of data exchanged by manufacturing resources providers.

A second vertical integration of information systems into MES and a step towards Industry 4.0 by usage of cloud computing solutions is the work of (Morariu, Borangiu, and Raileanu 2015) which considers the advantages of virtualization (e.g. improved resource utilization). Virtualization of the MES layer, as defined by the ISA-95 model, is implemented and described into details. Testing was done by deployment of the proposes solution on a local cloud and integrating a robotic cell with several Adept type robots. (Morariu et al.|2016) im- 
prove the previous research work by implementing and testing redundancy and scalability of virtualized MES.

(Yang, Zhang, and Chen 2016) highlights several issues found on the shop-floor which can generate manufacturing objects tracking inaccuracies in a MES. He proposes adoption of RFID systems and an on-line sequential extreme learning machine (OS-LEM) algorithm for data processing in order to deal with the lack of real-time data processing reliability and accuracy of manufacturing objects tracking.

A third vertical integration of a three-layers agent based MES in an enterprise is presented by (Cupek et al. 2016). The proposed MES was implemented in Microsoft Visual Studio Model-View-Controller having the goal to support production of customized short-series automotive parts. OPC UA is considered to achieve an efficient data exchange between manufacturing layers and resources towards a problem free integration and adaptability of the systems.

Another integration of information systems in a manufacturing enterprise is presented in (Iarovyi et al. 2016). This paper argues that efficient manufacturing operations can be achieved if data would have a common representation on all levels of information systems within a manufacturing enterprise. It is highlighted that most of available MES solutions have a centralized architecture which provides easy integration of hardware and software resources from the same vendor and have a reduced openness for integrating third party resources. A new MES concept called open knowledge development (OKD-MES) is proposed which uses the computational power of CPS for implementing semantics and ontologies to describe real word data into formalisms for enhanced usability. (Mohammed et al. 2018) describes how MES functions can be implemented in order to achieve increased flexibility in the interaction with vendor-independent web services using the OKD-MES framework.

\subsection{Summary and comparison of available MES}

A comparison related to the application fields of commercially available MES is presented in Table 1. This table contains a summary rather than a holistic overview of specific MES applications since results are based on public information available on specific databases, white papers and websites.

As one can observe, the provided MES solution are heavily depending on the application domain, hence the different vendors/solutions are specialized according mainly to these criteria. Also, the KPI are domain specific: (Apriso 2018) reports enhancements in the oil \& gas domain in terms of power consumption, flexibility in sensor infrastructure and time to decision making. For the electrical equipment producer Schneider Electric with the power of analytics with potential to drive $20 \%$ pull-through and $20 \%$ new connected services the revenue is expected to grow with $10 \%$. For the wood industry the IoT solutions based on azure delivered by the Accenture company helped to generate greater value from machines and cultivate a closer customer relationship positioning the wood company on the map of digital economy.

\subsection{MES key performance indicators}

Within enterprises, strategic business goals are translated into a set of quantifiable and measurable indicators. These indicators are referred as key performance indicators and they provide an insight of strategic goals fulfillment, understanding and improvement of manufacturing systems performances (Kang et al. 2016b).

To have a common reference in analyzing manufacturing systems, ISO20400 standard de- 


\begin{tabular}{|r|l|l|l|l|l|l|l|}
\hline \multicolumn{7}{|c|}{ MES comparison table } \\
\hline Manufacturer & ABB & Emerson & Siemens & Rockwell & Honeywell & Schneider & Delmia \\
\hline Product & Ability MOM & Syncade & Simatic IT & Factory Talk & BusinessFlex & Wonderware & Apriso \\
\hline Consumer Goods\&Retail & & & $\checkmark$ & $\checkmark$ & & $\checkmark$ & $\checkmark$ \\
\hline Eyewear & & & & & & & $\checkmark$ \\
\hline Food\& Beverage & $\checkmark$ & & $\checkmark$ & & & $\checkmark$ & $\checkmark$ \\
\hline Healt\&Beauty & & & $\checkmark$ & & & $\checkmark$ & $\checkmark$ \\
\hline Packaging & & & $\checkmark$ & & & & $\checkmark$ \\
\hline Tobacco & & & $\checkmark$ & & & $\checkmark$ & $\checkmark$ \\
\hline Brewing & & & $\checkmark$ & & & $\checkmark$ & $\checkmark$ \\
\hline Medical Devices & $\checkmark$ & & $\checkmark$ & & & $\checkmark$ & $\checkmark$ \\
\hline Pharmaceutical & & & & & & & $\checkmark$ \\
\hline Life Sciences Chemical & $\checkmark$ & $\checkmark$ & $\checkmark$ & $\checkmark$ & & $\checkmark$ & $\checkmark$ \\
\hline Oil\&Gas & & $\checkmark$ & $\checkmark$ & & $\checkmark$ & $\checkmark$ & \\
\hline Metal & & & $\checkmark$ & & $\checkmark$ & $\checkmark$ & \\
\hline Mining & $\checkmark$ & & $\checkmark$ & $\checkmark$ & & $\checkmark$ & \\
\hline Bio-fuels & & & $\checkmark$ & & & $\checkmark$ & \\
\hline Pulp\&paper & $\checkmark$ & & & & $\checkmark$ & & \\
\hline Auto & $\checkmark$ & & $\checkmark$ & & $\checkmark$ & & \\
\hline Aerospace & & & $\checkmark$ & & & & \\
\hline Ind. components & $\checkmark$ & & $\checkmark$ & $\checkmark$ & & & \\
\hline Wanufecesses \& WWT & $\checkmark$ & & $\checkmark$ & & & & $\checkmark$ \\
\hline Other & & & & & & \\
\hline & & & & & & $\checkmark$ & $\checkmark$ \\
\hline
\end{tabular}

Table 1.: Overview of the existing major MES solutions from different domains

fines a set of 34 KPIs for manufacturing operation management (ISO 22400:2014 2014). ISO20400 might be differently interpreted, thus (Fukuda and Patzke 2010) organizes the KPIs in 5 categories presented in table 2

Evaluation of KPI for the presented user story is not a major goal of this paper due to the fact that the developed workbench represents a small part from a production process: product testing phase and product dispatch phase. To have a real meaning, the KPIs can be objectively applied on a fully operating manufacturing system keeping in mind the business strategic objectives. Therefore only a subset of these KPI was in the focus of the analysis of this paper.

Nevertheless, four KPIs were selected to be displayed on the user graphical interface presented in Figure 5. Overall equipment efficiency (OEE) indicator is achieved because there is only one equipment (i.e. conveyor belt) that is not ready for operation. Utilization indicator is not met in this phase because there is no production order sent to the testing station. Quality indicator is met because there were no light bulb scraps during previous testing batch. An increase in worker productivity has been experienced since once Baxter cobot is taught how to act, the operator intervention in process related tasks is decreased. The waste ratio 


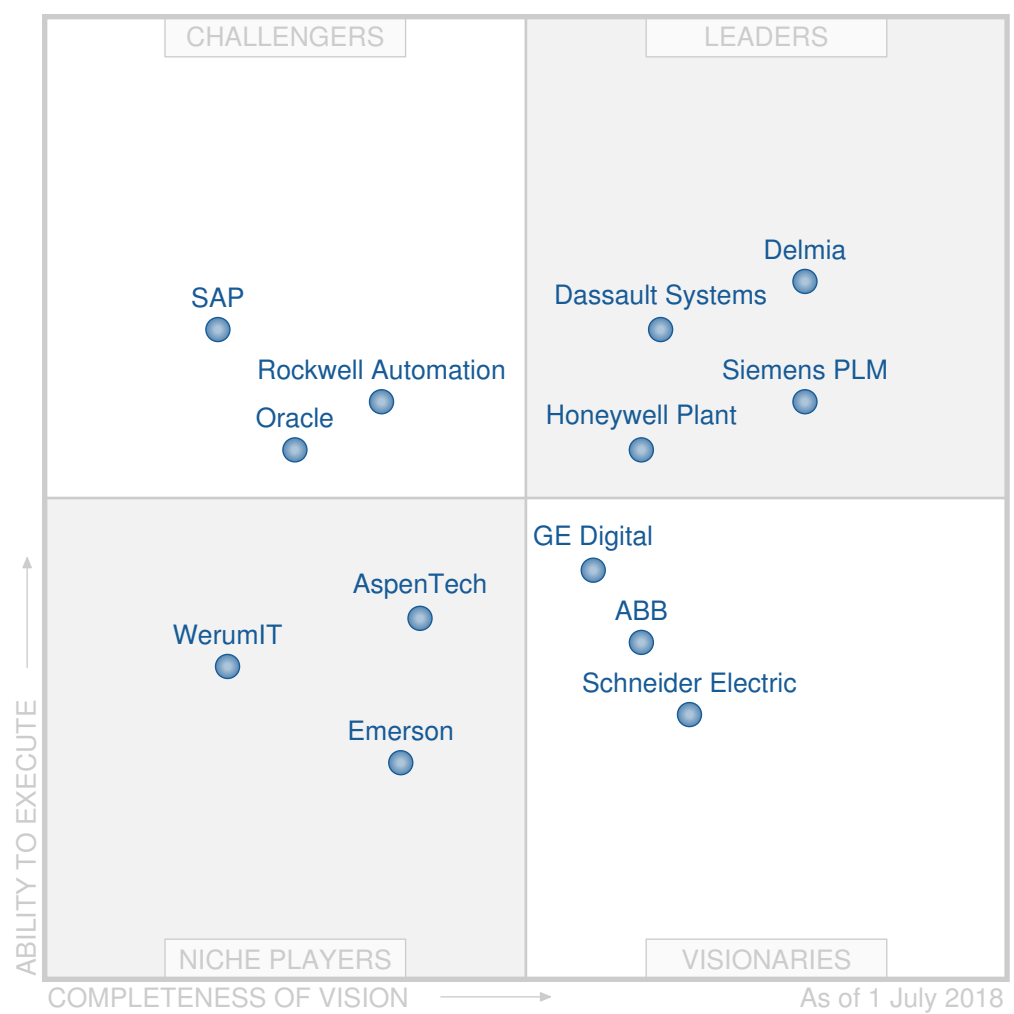

Figure 3.: Magic quadrant of the current MES commercial solutions

\begin{tabular}{|c|c|}
\hline Dimensions & KPI \\
\hline Performance & $\begin{array}{l}\text { Workers productivity, Allocation degree, Throughput, Allo- } \\
\text { cation efficiency, Overall equipment efficiency index, Net } \\
\text { equipment effectiveness index, Availability, Effectiveness }\end{array}$ \\
\hline Quality & $\begin{array}{l}\text { Quality rate, Preparation degree, Technical usage level, } \\
\text { Wastage degree, First pass yield, Waste ratio, Reworking } \\
\text { ration }\end{array}$ \\
\hline Capacity & $\begin{array}{l}\text { Machine Capacity index, Critical machine capability index, } \\
\text { Process capability index, Critical process capability index }\end{array}$ \\
\hline Environmental & $\begin{array}{l}\text { Environmental compatibility and emission ratio, Energy ra- } \\
\text { tio and ratio of used materials, Harmful substances, Haz- } \\
\text { ardous waste, Energy consumption, Comprehensive en- } \\
\text { ergy consumption }\end{array}$ \\
\hline Logistic & $\begin{array}{l}\text { Inventory handling and management, Inventory turns, Fin- } \\
\text { ished Good Rate, Integrated Goods Rate, Production Lost } \\
\text { Rate, Storage and Transportation Lost Rate, Load Rate }\end{array}$ \\
\hline
\end{tabular}

Table 2.: MES Key Performance Indicators 
was also mitigated, by appropriate robot workspace configuration the failure in the during light bulb testing phase was reduced, thus reducing the ratio of failed/wasted parts. Further implementation details related to the use case are presented in Section 3.

\section{MES Vertical Integration Use Case}

As a proof of concept for the MES vertical integration a custom/ROS package based cobot used in a commercial MES product is presented in the followings within a sorting application.

The dual-arm Baxter cobot is a collaborative type robot which means it can work side by side with people. From line loading to packing and unpacking Baxter has the flexibility to be deployed and re-deployed on tasks with minimum setup or integration cost. Baxter is characterized by having built in safety mechanism for humans. This built in safety is one of the main differences between the traditional industrial robot and this cobot. It takes just a few minutes to teach Baxter to pick up an object, in this case a light bulb and put it in the testing equipment. While the light bulb is in the testing equipment it checks if it lights up or not by observing the light bulb color, and picks the light bulb from the testing equipment and puts it to the right container according to the result of the test which is based on light bulb color. The robot as well as the workspace with the conveyor belt is presented in Figure 4. The application including the workspace setup, user interaction as well as the sorting process is captured on the video https: //youtu.be/2dhgU5n-JsE.

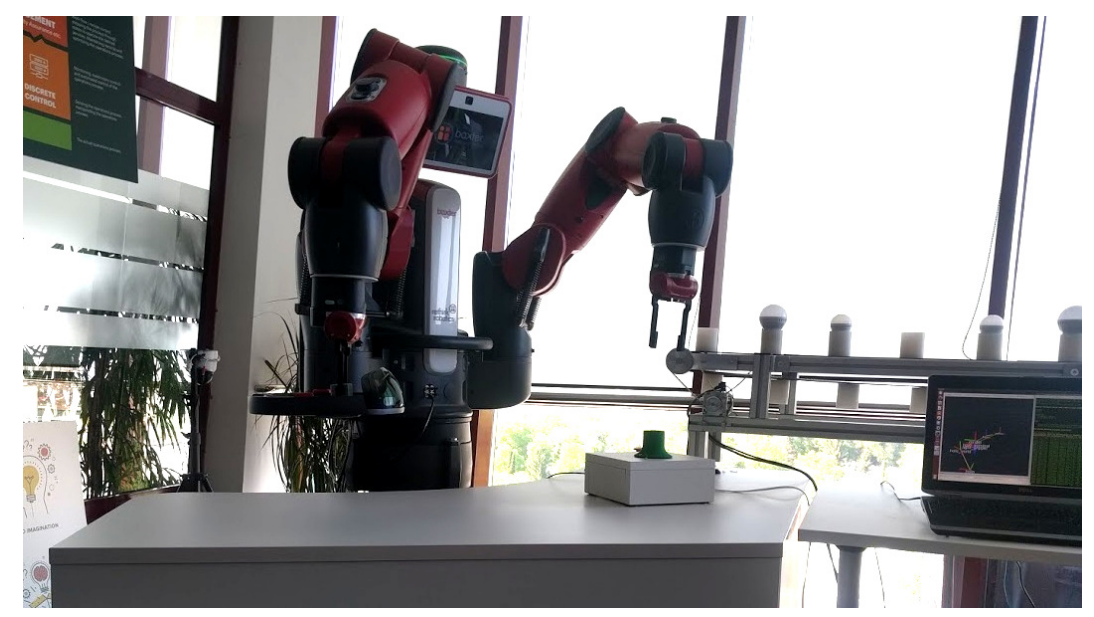

Figure 4.: Workspace of the robot in the initial phase

When a new order is received from the manufacturing enterprise end user interface the MES triggers the testing procedure for the required number of light bulbs. A conveyor belt controlled by a programmable logic controller (PLC) which is used to feed the cobot with light bulbs in the production. Close to the current use case is the one reported by (Iarovyi et al. 2016) based on open-knowledge driven MES for conveyor belt systems and the agent based approach described by (Cupek et al. 2016) for small batch production systems. Both applications have overlapping parts with the current work, but none of them is dealing with emerging solutions such as cobots, AGVs or AR visualization tools.

The PLC drives the conveyor belt which caries light bulbs until a proximity sensor identifies that the light bulb reached the pick position. The cobot handles the light bulbs from the conveyor belt towards a tester station. Light bulbs are placed in the tester station and the PLC is informed by cobot using Modbus communication protocol to start the testing procedure. The PLC is activating the coil of a relay for a user specified time-period and supplies the light 
bulb testing station with 230 VAC. Using its wrist cameras, the cobot identifies if the light bulb changes its state. Based on light bulb test results (valid or invalid) the cobot stores them in different locations accordingly to how it was taught by the operator.

The end user has a real-time high level overview of the whole process presented in a webpage fashion. Process information is also transmitted in real-time to all layers of the manufacturing enterprise. Detailed information are presented on local human machine interface (HMI) for manufacturing facility operators or to a supervisory control and data acquisition system (SCADA). Process related data is also sent to a cloud services provider where it can be further used for visualization or analytics and accessed from anywhere in the world with the right login credentials. The generic scheme of the involved manufacturing components as well as their connectivity is shown in Figure 1.

\subsection{Application overview}

The main aim of the application was to develop a flexible sorting application with a cobot, AGV and conveyor belt vertically integrated into MES system. The key process indicators in this case were related to the flexible reconfiguration of the workspace, equipment efficiency, equipment availability, number of scraps, number of sorted bulbs per unit time and the real time data analysis available for the operator.

In the first part a brief overview of the test-bench is presented, followed by a description ISA-95 layers implementation presented in Figure 3

The physical flow of this demo showcase is straightforward. The PLC is controlling the conveyor belt until a proximity sensor detects that the light bulb reached the picking position. The stepper motor from the conveyor belt is controlled by the motor driver, which starts the motor when a digital input is activated by PLC control logic. If the light bulb reached the pick position, the PLC stops the conveyor belt and triggers a function from cobot program to start the testing procedure. The communication between PLC and robot is done using Modbus TCP/IP protocol.

\subsubsection{ERP}

A graphic user interface presented in Figure 5 was developed on top of Apriso software infrastructure and integrated in the Business Planning and Logistics level of an ERP system to allow submitting production orders to the MES.

After introducing production specific information, one can submit the production order by pressing the Start button. The graphical UI displays information about the Production Order, Light Bulb, Produced Quantity and Target Quantity in a separate tab information related to Employee and Alerts are provided.

To provide a wide access on the top management level the graphical user interface was integrated into company dashboard developed under Siemens TeamCenter PLM.

\subsection{2. $M E S$}

The Light Bulb Factory MES application is composed from 13 entities developed in Process Builder. View and Action functions are associated with these entities. A View operation displays information for the user, an Action operation executes business logic or events in the background.

The standard functionalities of Apriso like Production, Quality, Inventory or Maintenance are built-in, and it is easy to extend, add new functionalities or in some cases to remove unnecessary features such as shown in Figure 6. 


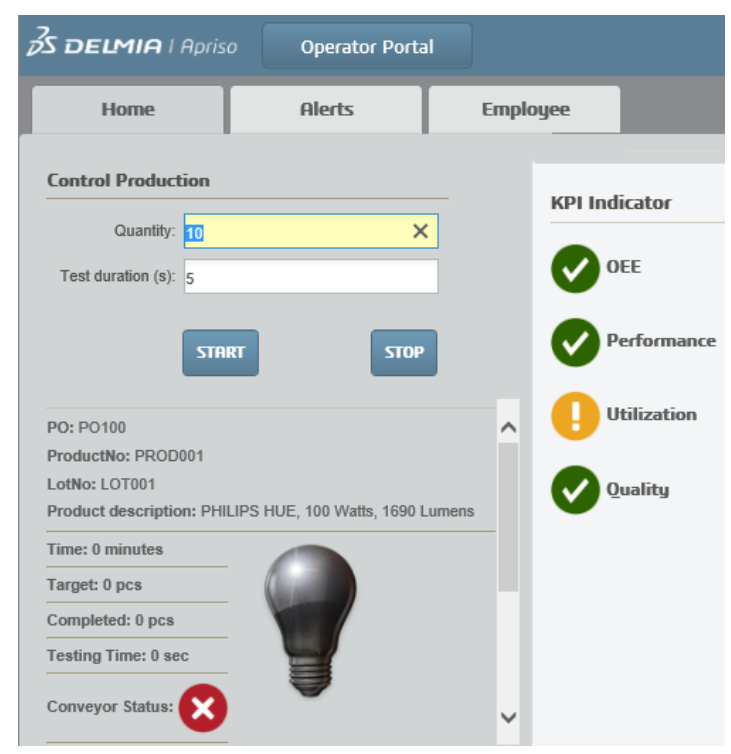

Figure 5.: Graphic user interface

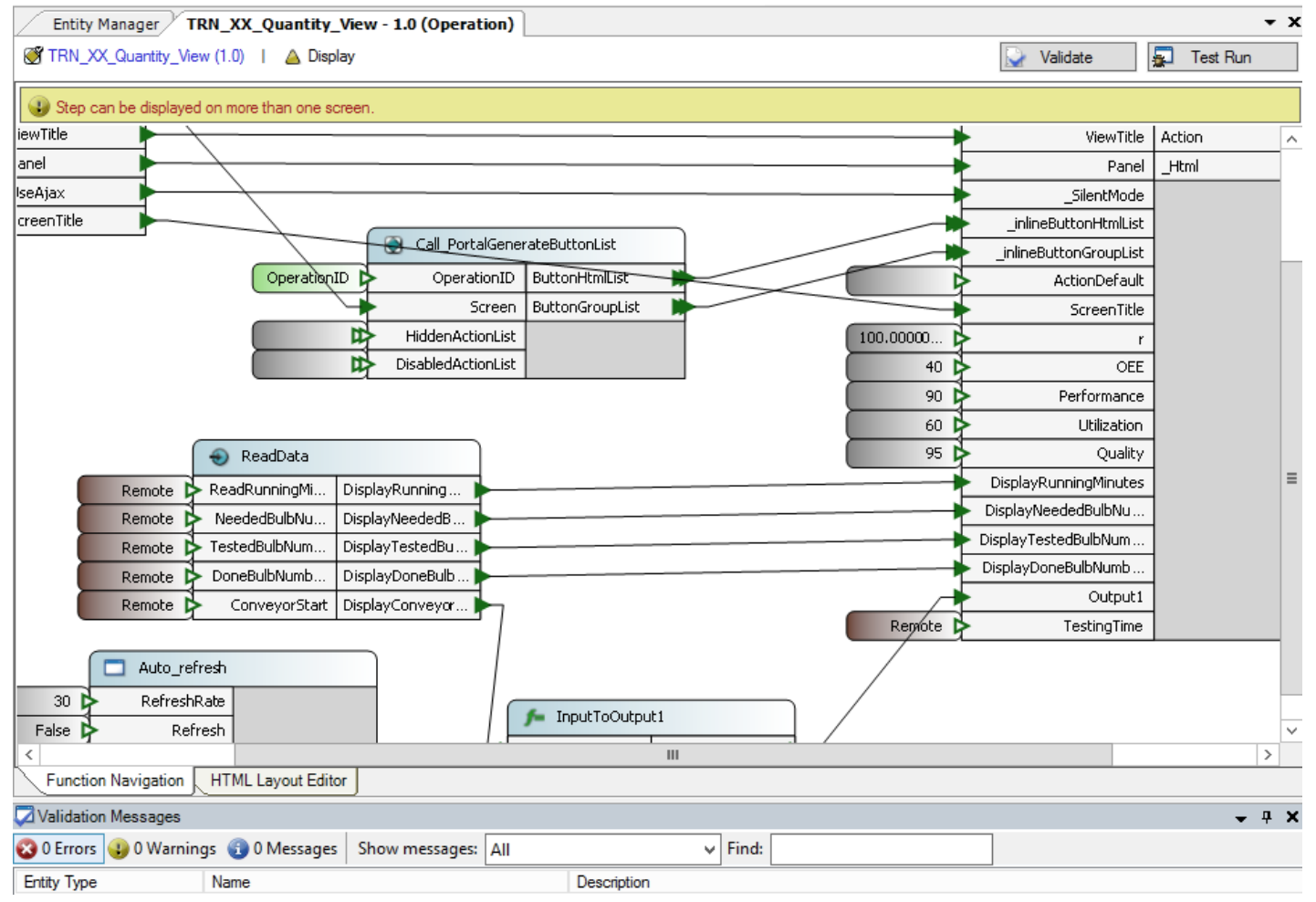

Figure 6.: MES components used in the application

In terms of MES components, this demo uses the following components with their appropriate functionalities which are presented in Figure 7 ;

- MS SQL Database: used to design SQL Objects, run test queries and troubleshoot data

- IIS \& MS SQL Services: web-server and database, core of DELMIA Apriso Suite

- Process Builder: Design Manufacturing Processes

- Global Process Manager: Bundle Apriso content (operation, file, object, configuration) 
into a package and easily transfer and manage its deployment in remote server

- Incoming File Monitor: Listen for inbound file and create a job out of them

- Job Executor: Run jobs, issues from file posting, from web services call, operation call

- Machine Integrator: Bridge to connect external systems.

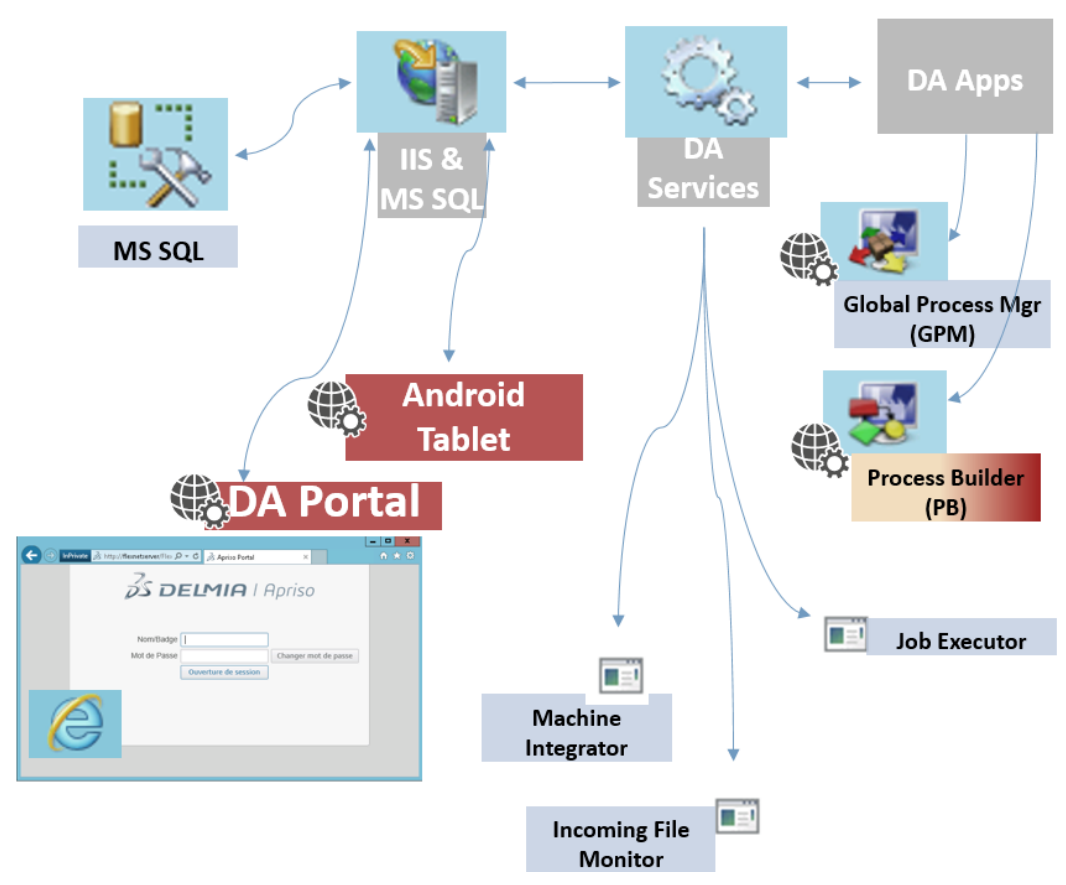

Figure 7.: MES components used in the application

Layout Editor is a functionality of IIS \& MS SQL Services used to design the user interface of operations in a convenient visual way, including positioning of screen elements. In addition, includes an HTML and CSS editor, so creating a flexible and user friendly screen or dashboard is straightforward.

In the developed test-bench the Apriso Machine Integrator is connected to the ERP using a MES database. ERP reads and writes specific data to the database and the logic within Apriso reacts to these triggers. Apriso Machine Integrator reads and writes data to the PLC controlling the shop-floor via an OPC server.

To have a good overview of a plant control in the context of MES is crucial. Traceability, time and labor, warehouse, maintenance and production, including the planning and other production related information are stored in a Microsoft SQL Database to which Apriso is connected.

Apriso Machine Integrator (Apriso 2018) supports standard communication protocols such as RS232 and OPC Server connection as well, and can be installed on many types of computers including servers, desktop computers and portable devices. With Apriso Machine Integrator (Apriso 2018) one can receive and send commands directly to the second level, the layer that controls and monitors the shop-floor.

\subsubsection{OPC layer}

Even if the OPC layer is not represented as an independent level in Figure 3, supporting efficient data exchange between with the third and the second levels is crucial. The OPC allows Windows programs to communicate with industrial hardware devices by means of this 
communication protocols.

MatrikonOPC Server was used to connect to the Siemens PLC from the second level into the MES. MatrikonOPC Sever comes with a diversity of industrial communication protocols to provide the required infrastructure to connect with PLCs from different vendors.

The S7TCP communication driver of the OPC Server is selected, the PLC IP address, rack number and the slot on the rack is configured as in the hardware configuration developed for the PLC in TIA Portal.

PLC tags can be accessed, tested and added manually or by means of XML files into the OPC server. Data match between PLC and MES is done by the OPC Sever. Data exchanged between OPC and PLC happens in a real-time communication. Further the OPC is connected to Apriso Machine Integrator.

\subsubsection{Control and monitor}

The set of equipment used in this application for control and monitor purposes are listed below:

- Siemens S7-1200 PLC

- Siemens KTP400 Touch Panel HMI

- Siemens IoT2040 intelligent industrial gateway

- Built-in Baxter controller

The control and monitoring of the process is done according to the state machine presented in Figure 8 In the first phase the workspace setup is done, i.e. the cobot is taking the reference position under the human operator guidance such as the positions where the bulbs should arrive, or the location of the quality check station.

The quality check part is controlled via a Modbus interfaced relay that enables high voltage to the bulb socket only during the quality testing phase. The testing command is sent from the cobot to the PLC, while the result of the testing is interpreted by the cobot based on the color/intensity of the bulb in the tester when supplied with energy. According to the test result the bulb is considered to be healthy/failed or of unknown type, and sorted accordingly. This information is send via Modbus TCPIP to the PLC and get by the OPCServer and fed to the MES.

Once a light bulb is tested and sorted the proximity sensor reads an empty socket on the conveyor belt and the conveyor is driven by the PLC until a new light bulb is detected by the sensor and a valid order is present as condition.

The sorting is done after the PackingTraining module was performed (see on Figure 8). In this module the operator teaches the robot in which box it should place the actual type of light bulb. The box position as well as the light bulb type associated to it by the user is send to the MES as well.

As the movement of the hands of cobot is easy to teach, the challenging part was to integrate a Modbus client in its software which is communicating with the PLC. When the light bulb arrives to the proximity sensor, the PLC modifies the value of a memory zone which is associated to Modbus server holding registers. On client side, in this case the Baxter, a Modbus client or listener is running and is checking if the holding registry value changed. So, when Baxter receives the information about the position of the light bulb, will start the picking procedure. When the light bulb arrives to the testing equipment, Baxter modifies the value of a holding register on PLC side. The PLC periodically checks all its associated holding registers. When a specific value is received the PLC knows that the light bulb is placed in testing equipment, so the power supply can be switched on.

The production contains a dispatch phase as well, in which an autonomously guided AGV 
is transporting the already sorted bulbs into the storage location. The starting signal to the $\mathrm{AGV}$ is sent from the MES, once one of the boxes contains the required number of valid bulbs. The production at this phase is continued only if a valid/new production batch is launched from the MES.

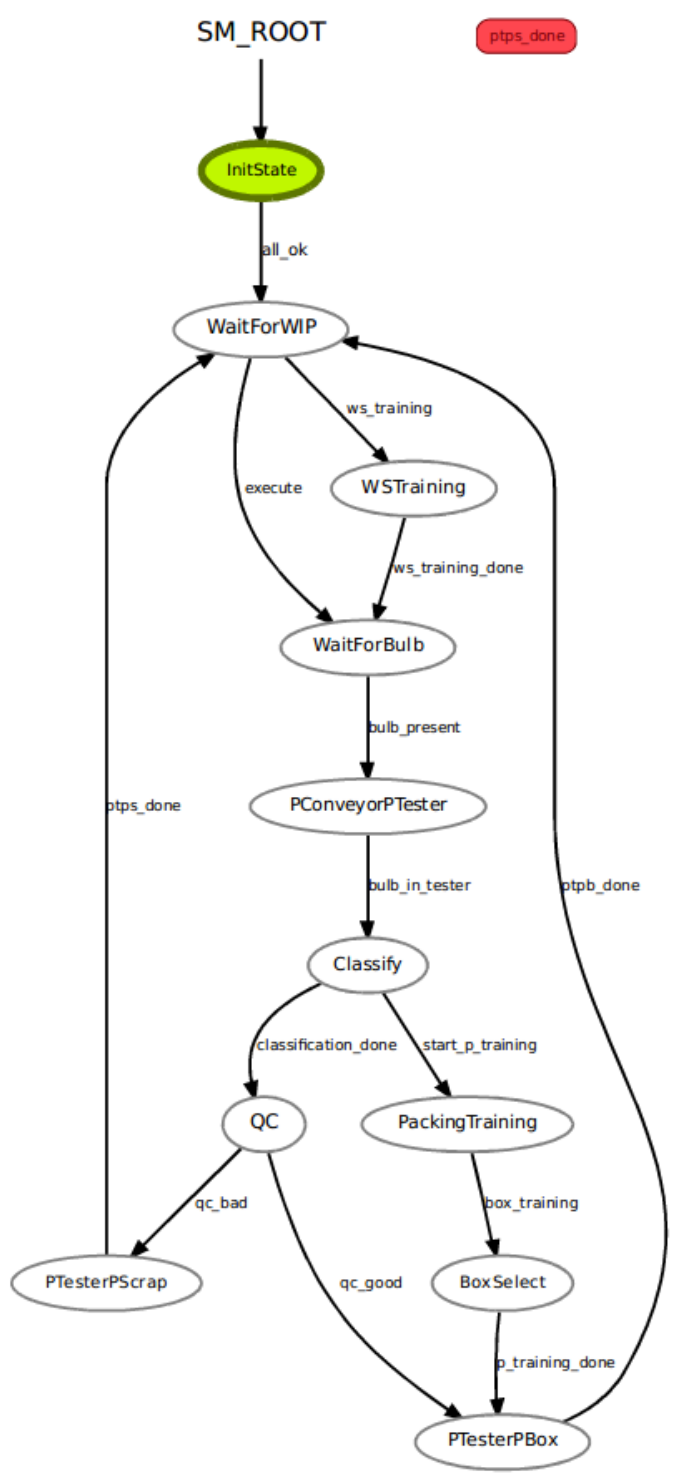

Figure 8.: State machine

On PLC side the standard MB_SERVER Modbus function is used. The function requires a database table to be attached, the holding registers are defined in this table. As additional inputs it has a tag used to connect and disconnect, basically to start or stop the server function, an ID and a Port number which the client must know in order to connect to Modbus Server.

The PLC supports up to eight Modbus server instances. One is used to create a Modbus server where the Baxter robot should connect to and another one is used to connect with the IoT2040 gateway. Since both Modbus instances used the Ethernet port of the PLC, they need to have different identification data. First Modbus server instance has the ID value configured to 2 and IP-PORT to 1200 and the second has the ID value configured to 3 and IP-PORT to 1201, see Figure 9 . The identification data is required for Modbus client to bridge a connection 
to the Modbus servers created in the PLC.

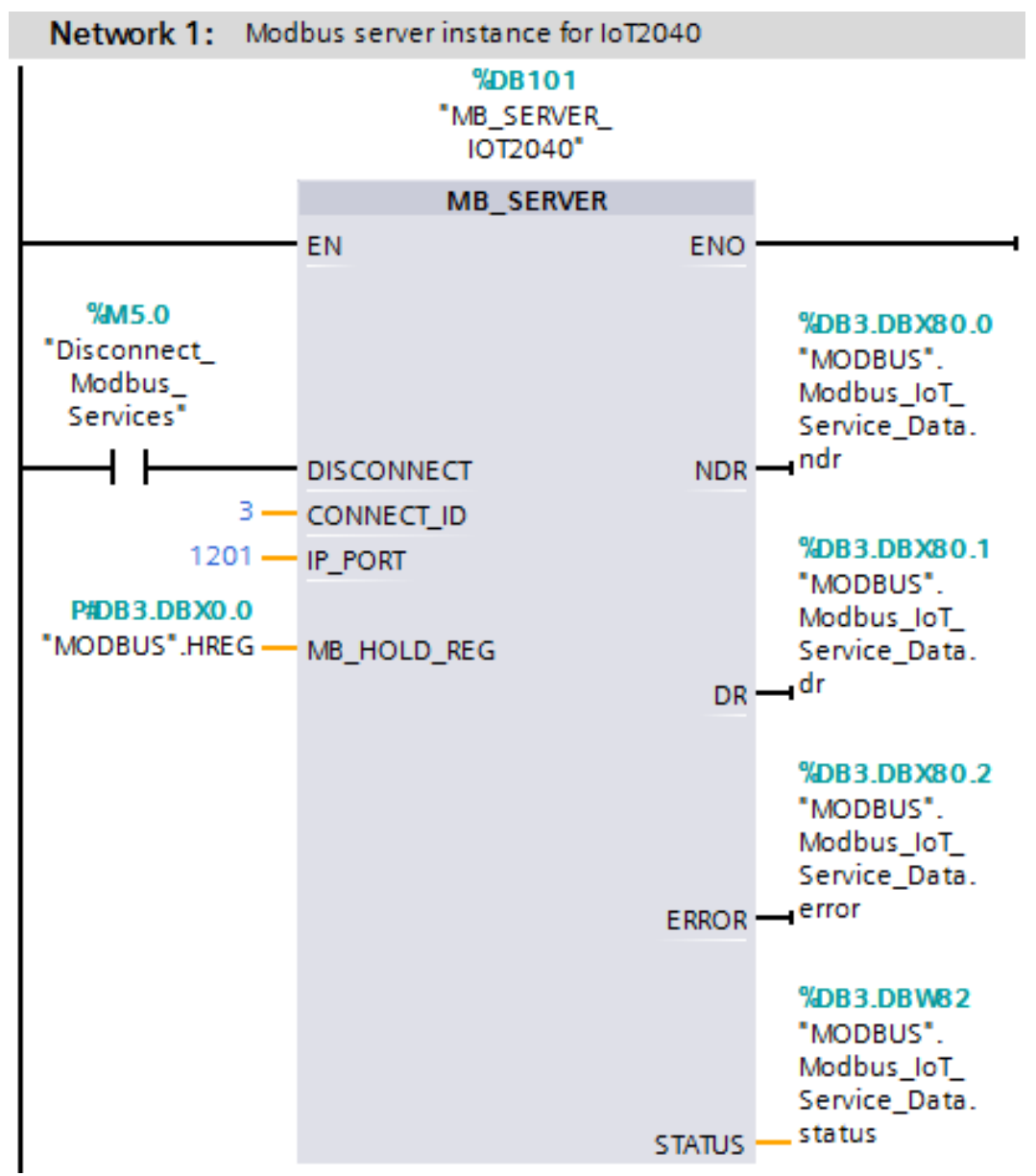

Figure 9.: Parametrization of IoT2040 Modbus server in PLC

The PLC program is relatively simple due to the fact that there are 2 digital outputs to be controlled: the conveyor belt and the relay for switching on and off the power for the testing equipment. PLC keeps track of functioning hours, ERP order, number of processed parts, number of scraps, the last maintenance date and other process related information which are displayed in the HMI panel on the shop-floor, see Figure 10 and for cloud application consider Figure 12

There are several signals interchanging in the Modbus interface related to the following actions in the work flow:

(1) Getting the position of the light bulb

(2) The robot is going to pick up the light bulb

(3) The robot is moving the light bulb from the conveyor belt to the tester

(4) The light bulb is in the tester

(5) Switching on and off the tester (for safety reasons)

(6) The number of light bulbs to be tested

(7) The packaging type of the order which is coming from PLM

An overview of the communication in the vertical integration is shown in Figure 11 


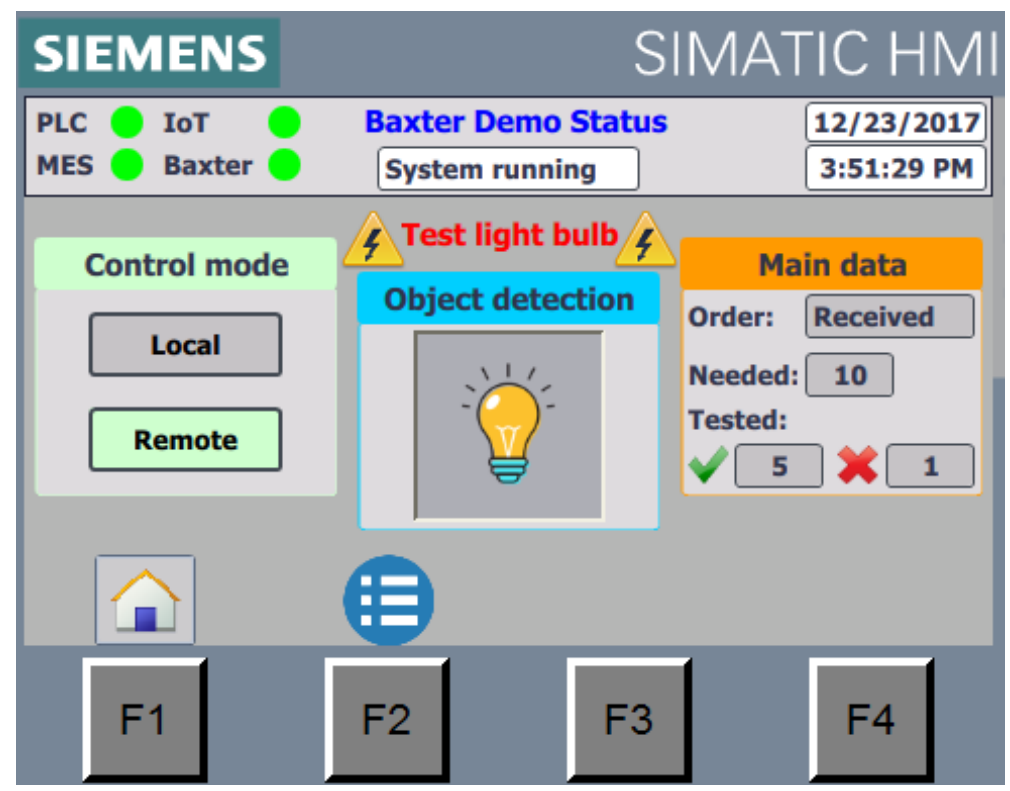

Figure 10.: Local process HMI

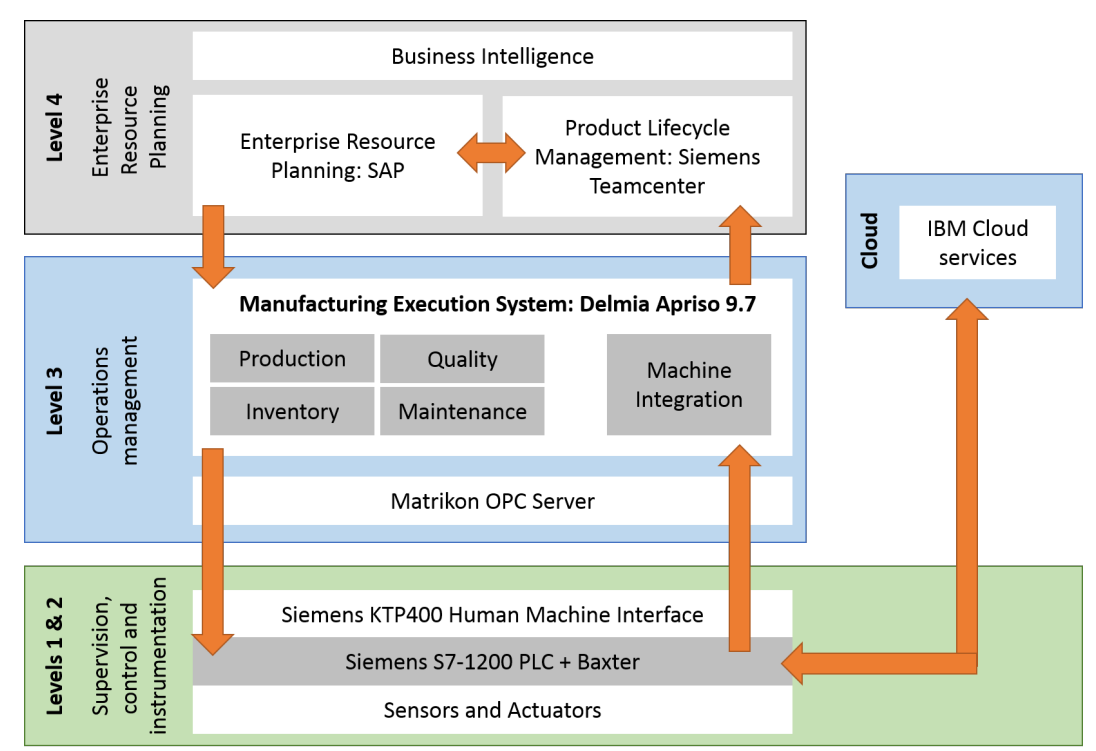

Figure 11.: Communication between different layers in the vertical integration

\subsubsection{Manufacturing resources and the shop-floor levels}

Manufacturing resources consists of level 1 with all the actuating and sensing equipment and Shop-floor consists of level 0 that represents the actual production process. On the manufacturing resources level there are: a light bulb sorting station composed by conveyor belt, quality check station, a dual arm cobot and a dispatching AGV unit.

The conveyor belt is custom made one designed for bulb transportation. The robot performing the sorting is a highly reconfigurable, collaborative robot, i.e. allows the direct interaction with a human operator during the production.

The set of equipment used in this application at this level are listed below:

- Stepper Motor and Driver 
- Baxter as a collaborative robot

- Conveyor belt

- Proximity sensor

- AGV from Turtlebot company

The control and monitoring of these devices is done by the previous level in the MES vertical hierarchy.

\subsubsection{Cloud}

The cloud should be seen as a parallel approach along the ERP and MES levels presented in figure 1. Connectivity with the cloud computing platform from IBM is achieved using an industrial gateway from Siemens, namely IoT2040.

The second Modbus TCP/IP connection configured in PLC and aforementioned in control and monitor subsection is configured to exchange data with an application developed on a programming environment running on the industrial gateway.

Node-RED (Node-RED 2018), a programming tool for development of IoT and cloud related applications, is one of the programming environment supported by this gateway.

On the application developed in the IoT2040 gateway runs a Modbus TCP/IP client which connects to the second Modbus TCP/IP Server that runs on the PLC to get process related data. Furthermore, using the Message Queuing Telemetry Transport (MQTT) machine-to-machine communication protocol an MQTT client running in the IoT2040 applications sends the data read from PLC to the HiveMQ MQTT broker which publishes the data to a subscribed client running in an application from an IBM Bluemix instance.

The application developed in cloud, using Node-RED, provides only monitoring functionalities for the process resources, see Figure 12 . The cloud application can be enhanced with control or other cloud related functionalities.

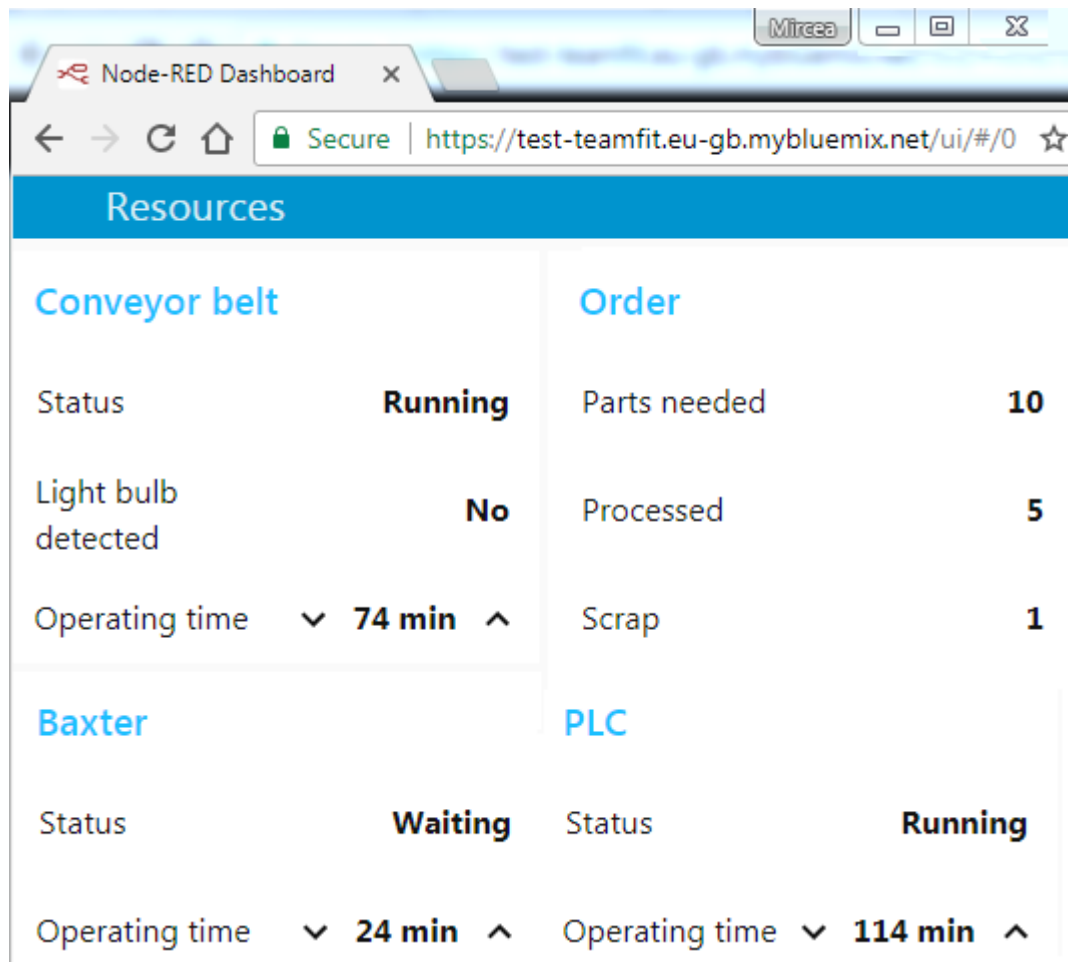

Figure 12.: IBM Bluemix cloud interface 


\subsection{Application summary}

In summary, the software modules and tools used to develop this application are the followings:

- Solidworks to design the CAD model for light bulb holders and tester box

- Siemens TIA Portal V13 for PLC and HMI programming

- node-RED for Siemens IoT2040 and IBM Bluemix

- Robot Operation System (ROS) for programming the Baxter cobot and the AGV

- DELMIA Apriso MES solution

- SAP ERP solution

- Teamcenter PLM solution

- IBM Bluemix as cloud service provider

The summary of the integration in one figure is shown in Figure 1, while the schematic overview of the system architecture is shown in Figure 13

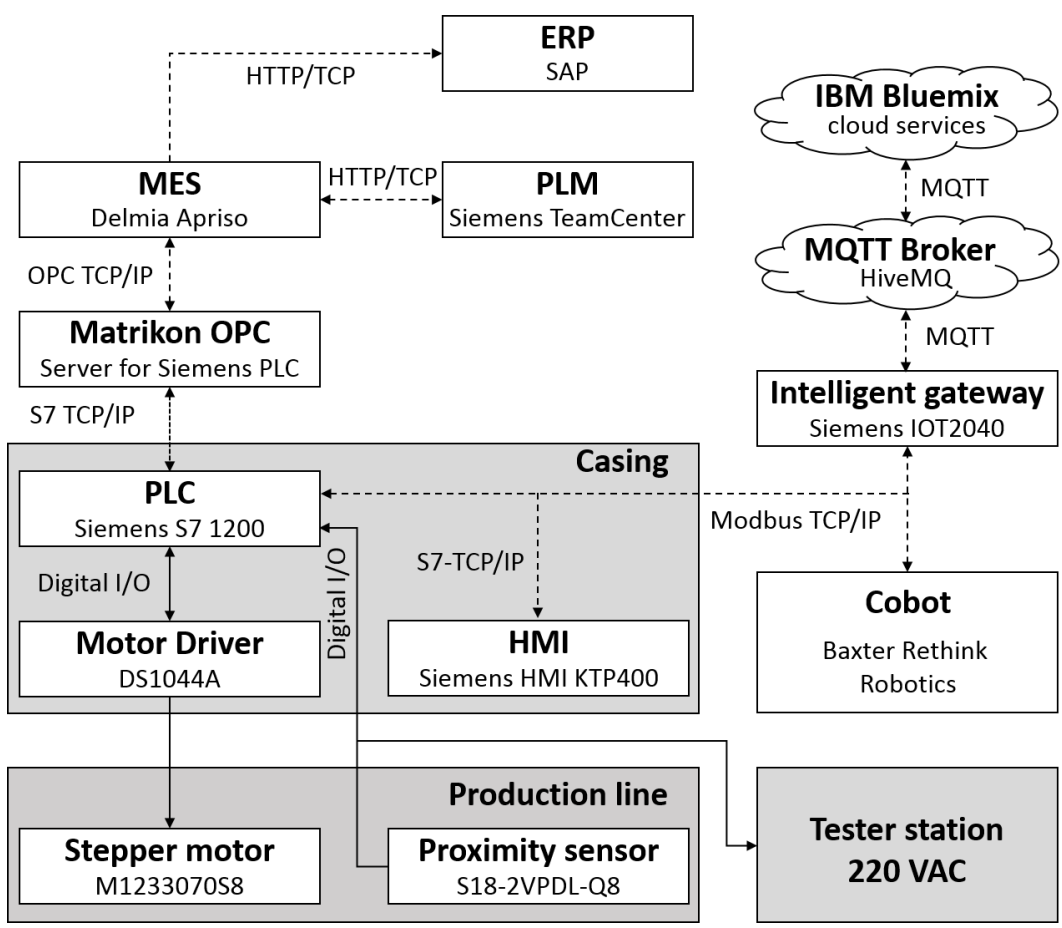

Figure 13.: Overview of a typical MES server network

\subsection{User experience}

To achieve a better user experience the user interface was designed using a special tool named Screen Framework(SF). SF has been introduced to address the up-to-date requirements of the user interface (UI) and to support the implementation of best practices for the UI and business logic (BL). SF facilitates the implementation of a rich UI that would normally require JavaScript and HTML or even C development.

In order to have a flexible and easy to understand interface, first the sequence diagram was designed such as this is visible in Figure 14. The user interaction is planned in two major phases: the workspace setup (organizing the physical locations of the pieces, boxes within 
the workspace of the robot) and the production setup (choosing the right location of the end products after sorting, quality check). The configurations for each user and setup is stored and made available for data analysis.

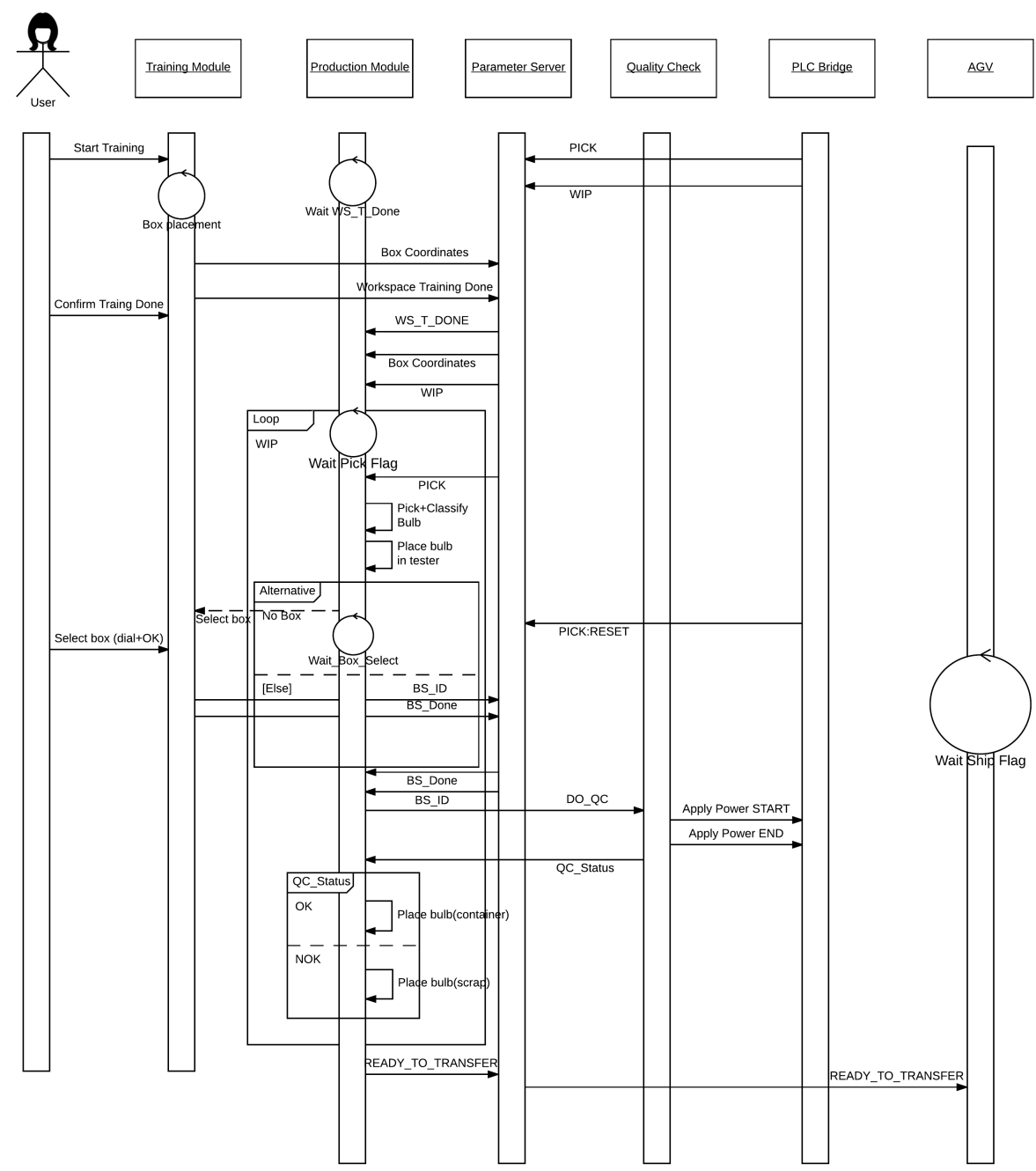

Figure 14.: Sequence diagram with the user interaction

The end result of the MES development is shown in the Figure 5. The Operator Dashboard displays information about the Production Order, Light Bulb, Produced Quantity, Target Quantity, the operator is able to start and stop the production, and a separate tab is created for Employee Maintenance and Alerts. The elements from this UI are both for the desktop and the tablet version for the end user.

In the near future within the MES is clearly expected a paradigm change for UI (Arica and Powell 2018): the visualization plays an important role both at lower and higher levels on the vertical scale. Currently the authors are already using some augmented reality related features (Blaga and Tamas 2018), which can be seen on also on this video: https://youtu.be/ BjXEL-VZbnw 


\section{Conclusion}

This paper highlights one of the most important trends in manufacturing environment: the most effective manufacturing system, Industry 4.0, characterized by a high level of digitalization of all layers in an enterprise. Manufacturing execution systems are used to fill the gap between shop-floor and enterprise resource planning solutions, increasing enterprise competitiveness and adaptability in real-time.

The lack of case studies and other MES related publications in academic environment to show the integration of manufacturing layers was the core enabler of the use case presented in this paper. The user case with the cobot, AGV, custom MES components are generic enough as it is based on the major trends found in the state-of-the-art. Within the state-of-the-art chapter, the available solutions and developments in the field of MES are presented. At the end of this chapter an overview of some of the most important commercially available MES implementations in different industries is presented.

The paper showed how shop floor equipment and technologies are interconnected with different manufacturing layers in such a way as to deliver a fully functional MES integration into the structure of an enterprise. The light bulb testing station, a part of the manufacturing system, was integrated into an ERP using a commercially available MES solution. With the current solution the client side challenges consisting of reactive decision making, rigid sensor infrastructure and custom HRI were mitigated. The production setup time was consequently reduced by more than $20 \%$, enhancing the overall equipment efficiency and making the production real-time data available to the operators. Emerging technologies such as IoT gateways, CPS, cloud computing and other related software were used to provide accessibility to testing station data from any place in the world using the MQTT communication protocol and broker.

Connecting a manufacturing processes to cloud computing provides new opportunities and research challenges. Data analytics and artificial intelligence can be applied to process and equipment related information on cloud platforms to better support preventive maintenance, scheduling or highlighting process optimizations.

Further work on this topic should consider the usage of PLCs or control units with integrated OPC servers to achieve a better interoperability between manufacturing resources and enterprise layers. Furthermore, the advantages of node-RED, such as the ease of use, should be taken into consideration in order to achieve cloud connectivity. For the emerging devices, smart glasses such as the ones with AR/VR capabilities for remote assistance purposes should be considered. The AGVs the cobots, next generation visualization devices, and custom cloud solutions are also expected to play an important role in the future manufacturing systems.

\section{Acknowledgment}

The authors would like to thank to their project colleagues (L. Tofalvi, L. Bagoly, P. Mago) and colleagues from the Technical University of Cluj-Napoca (C. Militaru, D. Mezei, L. Busoniu, S. Brad and D. Mitrea) their support and involvement in realizing this work. This work was supported by the Romanian National Authority for Scientific Research and Innovation under Grant number PN-III-P2-2.1-BG-2016-0140, Hungarian Research Fund, grant OTKA K 120367 and MTA Bolyai Scholarship. 


\section{References}

Apriso. 2018. http: //www.apriso.com/industries/

Arab-Mansour, Ikbal, Pierre Alain Millet, and Valérie Botta-Genoulaz. 2017. "A business repository enrichment process: A case study for manufacturing execution systems." Computers in Industry 89: $13-22$.

Arica, E, and D J Powell. 2018. "Status and future of manufacturing execution systems." 2017 IEEE International Conference on Industrial Engineering and Engineering Management (IEEEM) Dec: 2000-2004.

Barkmeyer, Ed, Peter Denno, Shaw Feng, Al Jones, and Evan Wallace. 1999. "NIST Response to MES Request for Information." .

Berger, Siegfried, and Matthias Wahrendorff. 2016. "Machine Dreams - Making the most of the Connected Industrial Workforce." Accenture 1-11.

Bevilacqua, Roberta, Elisa Felici, Fiorella Marcellini, Sebastian Glende, Susann Klemcke, Isabel Conrad, Raffaele Esposito, Filippo Cavallo, and Paolo Dario. 2015. "Robot-era project: preliminary results on the system usability." In Int. Conference of Design, User Experience, and Usability, 553561. Springer.

Blaga, Andreea, and Levente Tamas. 2018. “Augmented Reality for Digital Manufacturing.” In Control and Automation (MED), 2018 Mediterranean Conference of, 1020-1025. IEEE.

Canche, L., M. Ramrez, G. Jimenez, and A. Molina. 2004. "Manufacturing Execution Systems (MES) Based on Web Services Technology." IFAC Proceedings Volumes 37 (5): 135-140. 7th IFAC Symposium on Cost-Oriented Automation (COA 2004), Gatineau, Qubec, Canada, 6-9 June 2004.

Cheng, Fan-Tien, Chih-Feng Chang, and Shang-Lun Wu. 2004. "Development of holonic manufacturing execution systems.” Journal of Intelligent Manufacturing 15 (2): 253-267.

Cheng, Fan-Tien, Eric Shen, Jun-Yan Deng, and Kevin Nguyen. 1999. "Development of a system framework for the computer-integrated manufacturing execution system: A distributed objectoriented approach.” International Journal of Computer Integrated Manufacturing 12 (5): 384-402.

Choi, Byoung K., and Byung H. Kim. 2002. "MES (manufacturing execution system) architecture for FMS compatible to ERP (enterprise planning system)." International Journal of Computer Integrated Manufacturing 15 (3): 274-284.

CORBA. n.d. "Common Object Request Broker Architecture." Accessed 2018-06-22. http:// www.ois.com/Products/what-is-corba.html.

Cupek, Rafal, Adam Ziebinski, Lukasz Huczala, and Huseyin Erdogan. 2016. "Agent-based manufacturing execution systems for short-series production scheduling." Computers in Industry 82 (Supplement C): 245-258.

Dai, Qingyun, Runyang Zhong, George Q. Huang, Ting Qu, T. Zhang, and T. Y. Luo. 2012. "Radio frequency identification-enabled real-time manufacturing execution system: a case study in an automotive part manufacturer." International Journal of Computer Integrated Manufacturing 25 (1): 51-65.

De Ugarte, B. Saenz, A. Artiba, and R. Pellerin. 2009. "Manufacturing execution system - A literature review." Production Planning and Control 20 (6): 525-539.

Deuel, A.C. 1994. "The benefits of a manufacturing execution system for plantwide automation." ISA Transactions 33 (2): 113 - 124.

Fukuda, Y., and R. Patzke. 2010. "Standardization of Key Performance Indecator for manufacturing execution system.” In Proceedings of SICE Annual Conference 2010, Aug, 263-265.

Gonalves, Lourdes Andreo, and Rubens Maciel Filho. 2013. "Alcohol Production Process Modelling Based on Indicators using Transactional software, Industrial Automation and Manufacturing Execution Systems-MES.” Chemical Engineering Transactions 32: 1327-1332.

Groger, Christoph, Mark Hillmann, Friedemann Hahn, Bernhard Mitschang, and Engelbert Westkmper. 2013. “The Operational Process Dashboard for Manufacturing." Procedia CIRP 7 (Supplement C): 205-210. Forty Sixth CIRP Conference on Manufacturing Systems 2013.

Hawker, J. S. 1999. CIM Framework architecture and application models, 201-214. Boston, MA: Springer US.

Huang, Chin-Yin. 2002. "Distributed manufacturing execution systems: A workflow perspective." 
Journal of Intelligent Manufacturing 13 (6): 485-497.

Iarovyi, S., W. M. Mohammed, A. Lobov, B. R. Ferrer, and J. L. M. Lastra. 2016. "Cyber-Physical Systems for Open-Knowledge-Driven Manufacturing Execution Systems." Proceedings of the IEEE 104 (5): 1142-1154.

ISO 22400:2014. 2014. Automation systems and integration - Key performance indicators (KPIs) for manufacturing operations management. Standard. Geneva, CH: International Organization for Standardization.

Jung, Kiwook, SangSu Choi, Boonserm Kulvatunyou, Hyunbo Cho, and KC Morris. 2017. "A reference activity model for smart factory design and improvement." Production Planning \& Control 28 (2): 108-122.

Kagermann, Henning, Johannes Helbig, Ariane Hellinger, and Wolfgang Wahlster. 2013. Recommendations for Implementing the strategic initiative INDUSTRIE 4.0: securing the future of German manufacturing industry; final report of the Industrie 4.0 working group. Forschungsunion.

Kang, Hyoung Seok, Ju Yeon Lee, SangSu Choi, Hyun Kim, Jun Hee Park, Ji Yeon Son, Bo Hyun Kim, and Sang Do Noh. 2016a. "Smart manufacturing: Past research, present findings, and future directions." Int. Journal of Precision Engineering and Manufacturing-Green Technology 3 (1): 111128.

Kang, Ningxuan, Cong Zhao, Jingshan Li, and John A. Horst. 2016b. "A Hierarchical structure of key performance indicators for operation management and continuous improvement in production systems." International Journal of Production Research 54 (21): 6333-6350.

Ksksal, A., and E. Tekin. 2012. "Manufacturing Execution Through e-FACTORY System.” Procedia CIRP 3 (Supplement C): 591-596. 45th CIRP Conference on Manufacturing Systems 2012.

Lamparter, Steffen, Christoph Legat, Raffaello Lepratti, Joachim Scharnagl, and Lars Jordan. 2011. "Event-based Reactive Production Order Scheduling for Manufacturing Execution Systems*." IFAC Proceedings Volumes 44 (1): 2722-2730. 18th IFAC World Congress.

Lasi, Heiner. 2013. "Industrial Intelligence - A Business Intelligence-based Approach to Enhance Manufacturing Engineering in Industrial Companies.” Procedia CIRP 12 (Supplement C): 384-389. Eighth CIRP Conference on Intelligent Computation in Manufacturing Engineering.

Lee, Jay, Behrad Bagheri, and Hung-An Kao. 2015. "A cyber-physical systems architecture for Industry 4.0-based manufacturing systems." Manufacturing Letters 3: 18-23.

Li, Huiying, Xinfu Pang, Binglin Zheng, and Tianyou Chai. 2005. "The Architecture Of Manufacturing Execution System In Iron And Steel Enterprise.” IFAC Proceedings Volumes 38 (1): 181-186. 16th IFAC World Congress.

Lu, Yan, KC Morris, and Simon Frechette. 2016. "Current standards landscape for smart manufacturing systems." National Institute of Standards and Technology, NISTIR 8107.

Meier, Matthias, and Ursula Rauschecker. 2015. "Chapter 33 - Manufacturing Execution Systems for Micro-manufacturing." In Micromanufacturing Engineering and Technology (Second Edition), edited by Yi Qin, second edition ed., Micro and Nano Technologies, 749 - 773. Boston: William Andrew Publishing.

MESA. 1997. Execution-driven manufacturing management for competitive advantage: white paper 5.

Militaru, Cristian, Ady-Daniel Mezei, and Levente Tamas. 2017. "Lessons Learned from a Cobot Integration into MES." In ICRA - Recent Advances in Dynamics for Industrial Applications Workshop, Singapore.

Militaru, Cristian., Daniel Mezei, and Levente Tamas. 2016. “Object Handling in Cluttered Indoor Environment with a Mobile Manipulator.” In AQTR 2016: International Conference on Automation, Quality and Testing, Robotics, May.

Mohammed, Wael M., Borja Ramis Ferrer, Sergii Iarovyi, Elisa Negri, Luca Fumagalli, Andrei Lobov, and Jose L. Martinez Lastra. 2018. "Generic platform for manufacturing execution system functions in knowledge-driven manufacturing systems." International Journal of Computer Integrated Manufacturing 31 (3): 262-274.

Monostori, László. 2014. "Cyber-physical production systems: Roots, expectations and R\&D challenges." Procedia CIRP 17: 9-13.

Morariu, Octavian, Theodor Borangiu, and Silviu Raileanu. 2015. "vMES: Virtualization aware man- 
ufacturing execution system.” Computers in Industry 67 (Supplement C): 27-37.

Morariu, Octavian, Theodor Borangiu, Silviu Raileanu, and Cristina Morariu. 2016. "Redundancy and scalability for virtualized MES systems with programmable infrastructure." Computers in Industry 81 (Supplement C): 26-35. Emerging ICT concepts for smart, safe and sustainable industrial systems.

Naedele, Martin, Hong-Mei Chen, Rick Kazman, Yuanfang Cai, Lu Xiao, and Carlos V.A. Silva. 2015. "Manufacturing execution systems: A vision for managing software development." Journal of Systems and Software 101 (Supplement C): 59-68.

Nasarwanji, A, D Pearce, P Khoudian, and Worcester A. 2009. "The impact of manufacturing execution systems on labor overheads." In World Congress on Engineering Conference on, 734-737. IEEE.

Node-RED. 2018. https: / / flows.nodered.org/.

Panetto, Herv, and Arturo Molina. 2008. "Enterprise integration and interoperability in manufacturing systems: Trends and issues." Computers in Industry 59 (7): 641-646. Enterprise Integration and Interoperability in Manufacturing Systems.

Pascal, Blanc, and Pierre Demongodin, Isabeland Castagna. 2008. "A holonic approach for manufacturing execution system design: An industrial application." Engineering Applications of Artificial Intelligence 21 (3): 315-330.

Perzylo, Alexander, Nikhil Somani, Stefan Profanter, Ingmar Kessler, Markus Rickert, and Alois Knoll. 2016. "Intuitive instruction of industrial robots: Semantic process descriptions for small lot production.” In Intelligent Robots and Systems International Conference on, 2293-2300. IEEE.

RethinkRobotics. 2018. "Colaborative robots." Accessed 2018-06-17. http:// Www.rethinkrobotics.com/baxter/.

Roln, Milagros, and Ernesto Martnez. 2012. "Agent-based modeling and simulation of an autonomic manufacturing execution system." Computers in Industry 63 (1): 53-78.

Sauer, Olaf. 2010. Trends in Manufacturing Execution Systems, 685-693. Berlin, Heidelberg: Springer Berlin Heidelberg.

Simo, Jean Marcelo, Paulo Czar Stadzisz, and Grard Morel. 2006. "Manufacturing execution systems for customized production.” Journal of Materials Processing Technology 179 (1): 268-275. 3rd Brazilian Congress on Manufacturing Engineering.

Uhlmann, E, Eckhard Hohwieler, and Claudio Geisert. 2017. "Intelligent production systems in the era of Industrie 4.0-Changing mindsets and business models." 17: 5-24.

Uhlmann, Eckart, Abdelhakim Laghmouchi, Claudio Geisert, and Eckhard Hohwieler. 2017. "Decentralized Data Analytics for Maintenance in Industrie 4.0." Procedia Manufacturing 11: 1120 - 1126. 27th International Conference on Flexible Automation and Intelligent Manufacturing, FAIM2017, 27-30 June 2017, Modena, Italy.

Valckenaers, Paul, Hendrik Van Brussel, Paul Verstraete, Bart Saint Germain, and Hadeli. 2007. "Schedule execution in autonomic manufacturing execution systems." Journal of Manufacturing Systems 26 (2): 75-84. Distributed Control of Manufacturing Systems.

Van Brussel, Hendrik, Jo Wyns, Paul Valckenaers, Luc Bongaerts, and Patrick Peeters. 1998. "Reference architecture for holonic manufacturing systems: PROSA." Computers in Industry 37 (3): 255-274.

Verstraete, Paul, P. Valckenaers, H. Van Brussel, B. Saint Germain, K. Hadeli, and J. Van Belle. 2008. "Towards robust and efficient planning execution." Engineering Applications of Artificial Intelligence 21 (3): 304-314.

Wang, M. L., T. Qu, R. Y. Zhong, Q. Y. Dai, X. W. Zhang, and J. B. He. 2012. “A radio frequency identification-enabled real-time manufacturing execution system for one-of-a-kind production manufacturing: a case study in mould industry." International Journal of Computer Integrated Manufacturing 25 (1): 20-34.

Wang, Shiyong, Jiafu Wan, Daqiang Zhang, Di Li, and Chunhua Zhang. 2016. "Towards smart factory for Industry 4.0: A self-organized multi-agent system with big data based feedback and coordination." Computer Networks 101: 158-168.

Weyer, Stephan, Mathias Schmitt, Moritz Ohmer, and Dominic Gorecky. 2015. "Towards Industry 4.0-Standardization as the crucial challenge for highly modular, multi-vendor production systems." IFAC-PapersOnLine 48 (3): 579-584. 
Yang, Zhixin, Pengbo Zhang, and Lei Chen. 2016. "RFID-enabled indoor positioning method for a real-time manufacturing execution system using OS-ELM.” Neurocomputing 174 (Part A): 121133.

Zarte, Maximilian, Agnes Pechmann, Jeffrey Wermann, Frederik Gosewehr, and Armando Walter Colombo. 2016. "Building an Industry 4.0-compliant lab environment to demonstrate connectivity between shop floor and IT levels of an enterprise." In Industrial Electronics Society, IECON 2016-4 2nd Annual Conference of the IEEE, 6590-6595. IEEE.

Zhong, Ray Y., Q.Y. Dai, T. Qu, G.J. Hu, and George Q. Huang. 2013. "RFID-enabled real-time manufacturing execution system for mass-customization production." Robotics and Computer-Integrated Manufacturing 29 (2): 283-292. 